\title{
Comportamento de forrageamento e dieta de Polystictus superciliaris (Aves, Tyrannidae) no sudeste do Brasil
}

\author{
Diego Hoffmann ${ }^{1}$, Marcelo F. Vasconcelos ${ }^{2}$, Leonardo E. Lopes ${ }^{2} \&$ Marcos Rodrigues $^{1}$
}

1. Departamento de Zoologia, Instituto de Ciências Biológicas, Universidade Federal de Minas Gerais, C.P. 486, $31270-901$ Belo Horizonte, MG, Brasil. (diego_hoffmann@ @otmail.com).

2. Pós-graduação em Ecologia, Conservação e Manejo de Vida Silvestre, Instituto de Ciências Biológicas, Universidade Federal de Minas Gerais, C.P. 486, 31270-901 Belo Horizonte, Minas Gerais, Brasil.

\begin{abstract}
Foraging behavior and diet of Polystictus superciliaris (Aves, Tyrannidae) in southeastern Brazil. The present article describes the foraging behavior and diet of Polystictus superciliaris (Wied, 1831), a poorly known species restricted to eastern Brazilian mountains. The work was carried out from April to December 2005 in the Serra do Rola Moça State Park, Nova Lima, Minas Gerais $\left(20^{\circ} 03^{\prime} \mathrm{S}, 44^{\circ} 00^{\prime} \mathrm{W}\right)$. For each foraging bout we recorded the attack behavior, attack direction, substrate, height of foraging substrate, and habitat type where the attack occurred. The species diet was determined through direct field observations and by analyzing the stomach contents of 16 individuals. Polystictus superciliaris uses mainly "canga couraçada" (93.3\%), and leaves (69.1\%) of the most abundant plants of the area as substrate for the capture of preys. The species is generalist in relation to attack behavior. Analysis of stomach contents revealed the exclusive presence of arthropods. Field observations confirmed the exclusive insectivory of $P$. superciliaris, which was not observed consuming fruits or other vegetable matter.
\end{abstract}

KEYWORDS. Espinhaço Range, foraging behavior, stomach contents, diet, canga.

RESUMO. O presente artigo descreve o comportamento de forrageamento e a dieta de Polystictus superciliaris (Wied, 1831), espécie pouco conhecida e de distribuição geográfica restrita às montanhas do leste do Brasil. Este trabalho foi realizado entre abril e dezembro

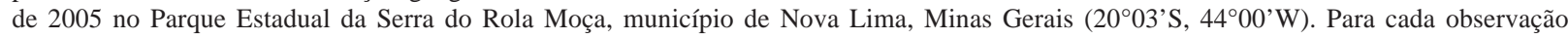
de forrageamento foram registrados os comportamentos de ataque, a direção e o substrato do ataque, a altura do substrato de forrageamento e a fitofisionomia onde o ataque ocorreu. Para a determinação da dieta da espécie foram feitas observações diretas de campo, sendo também analisado o conteúdo estomacal de 16 indivíduos. Polystictus superciliaris usa preferencialmente a canga couraçada (93,3\%), tendo como substrato principal as folhas $(69,1 \%)$ das plantas mais abundantes na área. A espécie é generalista quanto ao comportamento de ataque. A análise de conteúdos estomacais revelou a presença exclusiva de artrópodes. As observações confirmaram o caráter insetívoro de $P$. superciliaris, que não foi observado consumindo frutos ou outro material de origem vegetal.

PALAVRAS-CHAVE. Cadeia do Espinhaço, comportamento de forrageamento, conteúdo estomacal, dieta, canga.

Os tiranídeos ou papa-moscas (Passeriformes: Tyrannidae) formam um dos grupos de aves da Região Neotropical mais diversificados em termos de número de espécies (Sibley \& Monroe JR., 1990). As espécies de tiranídeos se adaptaram a uma enorme variedade de ambientes e nichos ecológicos e apresentam diversificação morfológica e uma riqueza de repertório comportamental sem paralelo entre os passeriformes suboscines (TRAYLOR \& FitzPatrick, 1982; Sick, 1997). Toda essa diversidade de forma e comportamento faz com que o grupo seja ideal para estudos filogenéticos (LANYON, 1986, 1988; BIRDSLEY, 2002).

Detalhes sobre o comportamento de forrageamento e dieta freqüentemente fornecem subsídios para inferências sobre a origem e evolução dos distintos subgrupos (subfamílias) de Tyrannidae (FitzPatrick, 2004). Dessa forma, tais aspectos da biologia dos Tyrannidae têm sido objeto de vários estudos (Sкuтch, 1960; Sмітн, 1971; FitzPatrick, 1980, 1985; Parker, 1984; Gabriel \& Pizo, 2005; LOPES, 2005).

A recente filogenia proposta para os Tyrannidae, baseada em caracteres morfológicos e comportamentais, mostra Polystictus como grupo irmão de Pseudocolopteryx (BIRDSLEY, 2002). Entretanto, a posição sistemática desse grupo ainda não se encontra bem resolvida, pois não há informações sobre o comportamento de muitas das espécies consideradas nas análises.

Polystictus superciliaris (Wied, 1831) é encontrada desde o Morro do Chapéu, no centro da Bahia, até a Serra da Bocaina, no norte de São Paulo (VAsconcelos et al., 2003). A espécie ocorre em altitudes entre 900 e $1.950 \mathrm{~m}$, onde habita os campos rupestres, campos cerrados e campos de altitude (SiCK, 1997; VASCONCELOS et al., 2003).

Informações sobre o comportamento de forrageamento de Polystictus superciliaris são escassas, derivadas de algumas poucas observações ocasionais (RIDGELY \& TUDOR, 1994; VASCONCELOS, 1999). Já sobre a dieta, cujo conhecimento é fundamental para se entender a história natural e desenvolver estratégias de conservação (BARTHOLOMEw, 1986; LoPES et al., 2005), tais informações são praticamente inexistentes, havendo apenas algumas observações pontuais (VASCONCELOS, 1999). O presente artigo descreve o comportamento de forrageamento e a dieta de $P$. superciliaris, uma espécie de distribuição geográfica restrita às montanhas do leste do Brasil (VAsConcelos et al., 2003). Este trabalho também representa uma contribuição que pode auxiliar na resolução da complexa filogenia dos Tyrannidae.

\section{MATERIAL E MÉTODOS}

Este trabalho foi realizado no Parque Estadual da Serra do Rola Moça, porção sul da Cadeia do Espinhaço, localizado entre os municípios de Belo Horizonte, Nova 
Lima, Brumadinho e Ibirité, Minas Gerais, Brasil (20 $03^{\circ} \mathrm{S}$, $\left.44^{\circ} 00^{\prime} \mathrm{W}\right)$. O parque possui uma área de $3.942 \mathrm{ha}$.

A região apresenta clima do tipo mesotérmico (Cwb de Köpen) com regime de precipitação bem definido, com verões chuvosos e invernos secos (NIMER, 1979). A área recebe uma precipitação anual de $1.735 \mathrm{~mm}$, a maior parte concentrada entre os meses de novembro e março (INMET, 2005).

A área de estudo é composta predominantemente por campos ferruginosos, estando situada em uma zona de transição entre o Cerrado e a Mata Atlântica (AB'S SABER, 1977). O substrato local (canga) é rico em ferro e a cobertura vegetal varia conforme o seu grau de agregação. Quando a canga forma uma couraça, ou laje, ela é denominada canga couraçada e apresenta uma cobertura vegetal com a presença de plantas que têm suas raízes desenvolvidas principalmente em pequenas fendas. Nestes locais as gramíneas são praticamente inexistentes. Quando o substrato se mostra fragmentado, tem-se a canga nodular, que é mais penetrável e apresenta cobertura vegetal dominada por canelas-de-ema (Velloziaceae) e gramíneas, que recobrem totalmente o substrato (RizzinI, 1979). Existem poucos estudos específicos sobre a fauna e flora desse tipo de ambiente, que se encontra sob grande pressão antrópica devido à expansão urbana, extração ilegal de plantas ornamentais (orquídeas e bromélias), trânsito de veículos off-road, mineração e turismo "ecológico" (VINCENT et al., 2002). O Quadrilátero Ferrífero, que engloba a área de estudos, é considerado de importância biológica especial (a mais alta categoria) em Minas Gerais devido ao elevado endemismo de anfíbios e plantas, à alta riqueza de vertebrados e à presença dos campos ferruginosos, um ambiente único no estado (DrumMOND et al., 2005).

As observações foram realizadas entre abril e dezembro de 2005 e todas as medidas de distância e altura apresentadas foram estimadas visualmente. Para cada observação de forrageamento foram registrados: o comportamento de ataque segundo REMSEN \& ROBINSON (1990) e convertido para a nomenclatura proposta por FITZPATRICK (1980) (para efeito de comparação); a direção do ataque (horizontal, vertical acima, vertical abaixo, diagonal acima e diagonal abaixo); o substrato de ataque (folha, ramo, flor, ar, solo); a altura do substrato de forrageamento $(0,1-1 \mathrm{~m} ; 1,1-2 \mathrm{~m} ; 2,1-3 \mathrm{~m} ; 3,1-4 \mathrm{~m} ; 4,1-5$ $\mathrm{m}$ ), e a fitofisionomia onde o ataque ocorreu (campo ferruginoso sobre canga couraçada ou sobre canga nodular). Os diferentes tipos de comportamento de ataque reconhecidos por REMSEN \& ROBINSON (1990) foram grafados em português, seguindo a proposta de VolPATO \& Mendonça-Lima (2002), sendo mantida a nomenclatura em língua inglesa para os comportamentos reconhecidos por FitZPATRICK (1980).

Os indivíduos foram observados com o auxílio de binóculos, sendo o comportamento de forrageamento registrado a partir de 5 min após a primeira visualização do indivíduo, a fim de que a ave se acostumasse com o pesquisador. A partir de então foi registrado um novo comportamento a cada intervalo de $5 \mathrm{~min}$, o que permitia, devido à grande mobilidade da espécie, consideráveis deslocamentos entre os pontos de amostragem. Foram obtidos registros de forrageamento para cerca de 20 indivíduos, sendo raramente tomados mais do que cinco registros para cada indivíduo em um único dia. Tais procedimentos contribuíram para uma substancial redução na autocorrelação das amostras, que poderia levar a uma estimativa imprecisa das variáveis comportamentais medidas neste estudo (HEJL et al., 1990).

Para a determinação da dieta da espécie, foram realizadas observações diretas no campo com o auxílio de binóculos. Adicionalmente, analisou-se o conteúdo estomacal das carcaças de 16 exemplares depositados na coleção ornitológica do Departamento de Zoologia da Universidade Federal de Minas Gerais, Belo Horizonte (DZUFMG). Os fragmentos de artrópodes encontrados foram identificados com o auxílio de livros-texto de entomologia (e. g. Borror et al., 1989). Depois de identificados, os fragmentos foram contados a fim de se estimar o número de presas ingeridas. Os insetos foram agrupados em ordens e o restante dos artrópodes em classes. Detalhes sobre os métodos utilizados para a identificação dos conteúdos estomacais são apresentados em Lopes et al. (2005).

\section{RESULTADOS}

Foram obtidos 149 registros de forrageamento de $P$. superciliaris, que se utilizou principalmente dos campos ferruginosos sobre canga couraçada $(93,3 \%)$, os quais recobrem $73,6 \%$ de toda a área de estudo, seguido da canga nodular $(6,7 \%)$, que recobre $18,9 \%$ da área. Como substrato de forrageamento foram utilizadas principalmente as folhas $(69,1 \%)$ e ramos $(26,8 \%)$, seguidos pelas flores $(2,0 \%)$, ar $(1,3 \%)$ e liquens $(0,8 \%)$. As principais espécies de plantas utilizadas como substrato de forrageamento foram Lychnophora ericoides (Asteraceae) $(21,5 \%)$ e Mimosa calodendron Ex. Benth. (Mimosaceae) $(13,4 \%)$ (Fig. 1). A altura predominante do substrato de forrageamento foi $0,1-1 \mathrm{~m}$ (81,9\%), seguida do intervalo 1,1-2 m (14,8\%) (Fig. 2).

Polystictus superciliaris utilizou oito manobras de forrageamento, conforme REMSEN \& RoBINSON (1990), com predomínio de investir-estolar $(30,9 \%)$, investir-atingir $(22,1 \%)$ e alcançar $(19,5 \%)$ (Fig. 3). P. superciliaris apresentou quatro manobras de forrageamento de acordo com a nomenclatura de FitZPATRICK (1980), com predomínio de "simple perch-gleaning" (47\%) e "upward striking" $(36,2 \%)$ (Fig. 4). A direção predominante da investida foi a horizontal $(40,9 \%)$, seguida pela diagonal acima $(35,6 \%)$ (Fig. 5). Geralmente P. superciliaris forrageou em pares $(75,2 \%)$ e, em menor escala, $(24,8 \%)$ solitariamente. Em nenhuma ocasião foi registrada associação a bandos mistos ou monoespecíficos.

A alimentação é composta exclusivamente por pequenos artrópodes, não tendo sido encontrados itens de origem vegetal. A análise dos conteúdos estomacais revelou principalmente o consumo de Coleoptera e Hemiptera (Tab. I), geralmente com comprimento inferior a $10 \mathrm{~mm}$. Durante as observações de campo, somente os artrópodes de maior porte puderam ser identificados, sendo registrada a captura de quatro lagartas (Lepidoptera), três borboletas (Lepidoptera) e quatro gafanhotos (Orthoptera). Após a captura de uma presa um pouco maior, os indivíduos apresentavam o mesmo comportamento, batendo-a no ramo mais próximo algumas vezes para, logo após, engolirem-na. 
Tabela I. Número de presas encontrado em 16 estômagos de Polystictus superciliaris (Wied, 1831) entre abril e dezembro de 2005 no Parque Estadual da Serra do Rola Moça, Minas Gerais, Brasil. A primeira coluna indica o número de tombo de cada exemplar na Coleção do Departamento de Zoologia da Universidade Federal de Minas Gerais (DZUFMG).

\begin{tabular}{|c|c|c|c|c|c|c|c|c|c|c|c|c|}
\hline Número & Orthoptera & Mantodea & Hemiptera & Homoptera & Coleoptera & Diptera & $\begin{array}{l}\text { Lepidoptera } \\
\text { adulto }\end{array}$ & $\begin{array}{c}\text { Lepidoptera } \\
\text { larva }\end{array}$ & Hymemoptera & $\begin{array}{l}\text { Larva de } \\
\text { inseto }\end{array}$ & Aranea & $\begin{array}{l}\text { Total de } \\
\text { artrópodes }\end{array}$ \\
\hline 4509 & & & 2 & & 4 & & 1 & & 1 & 1 & & 9 \\
\hline 3035 & & & 2 & & 2 & & & & & & & 4 \\
\hline 3036 & & & & 2 & 9 & & 1 & 1 & 4 & & & 17 \\
\hline 2830 & & & 3 & & 4 & & & 1 & 1 & & 1 & 10 \\
\hline 4046 & 1 & & 3 & & & & & & & & & 4 \\
\hline 4048 & & & & & 1 & & & & & & & 1 \\
\hline 4047 & & & 1 & & & 1 & & & & & & 2 \\
\hline 3043 & & & 1 & & 2 & & & 3 & 2 & & & 8 \\
\hline 2831 & & & & & 1 & & & & 2 & & & 3 \\
\hline 4173 & & & 7 & & & & 1 & 1 & & & & 9 \\
\hline 4592 & 1 & & 1 & 2 & 1 & 2 & 1 & & & & 2 & 10 \\
\hline 3037 & & & 3 & & 2 & 1 & & 2 & & & & 8 \\
\hline 2797 & & & 1 & & 3 & & & & & & & 4 \\
\hline 2832 & 1 & & 1 & & 7 & & & & & & 2 & 11 \\
\hline 3034 & & & & & & & & & & & 1 & 1 \\
\hline 4049 & & 1 & 7 & 2 & 2 & & & & & & & 12 \\
\hline Total & 3 & 1 & 32 & 6 & 38 & 4 & 4 & 8 & 10 & 1 & 6 & 113 \\
\hline
\end{tabular}

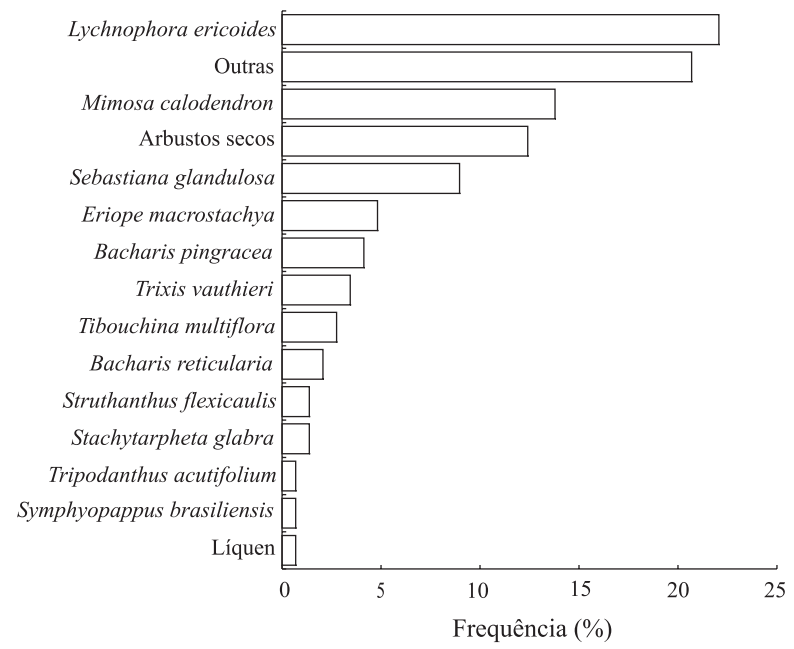

Fig. 1. Freqüência de observações de Polystictus superciliaris (Wied, 1831) em diferentes espécies de plantas utilizadas em atividade de forrageamento entre abril e dezembro de 2005 no Parque Estadual da Serra do Rola Moça, Minas Gerais, Brasil.

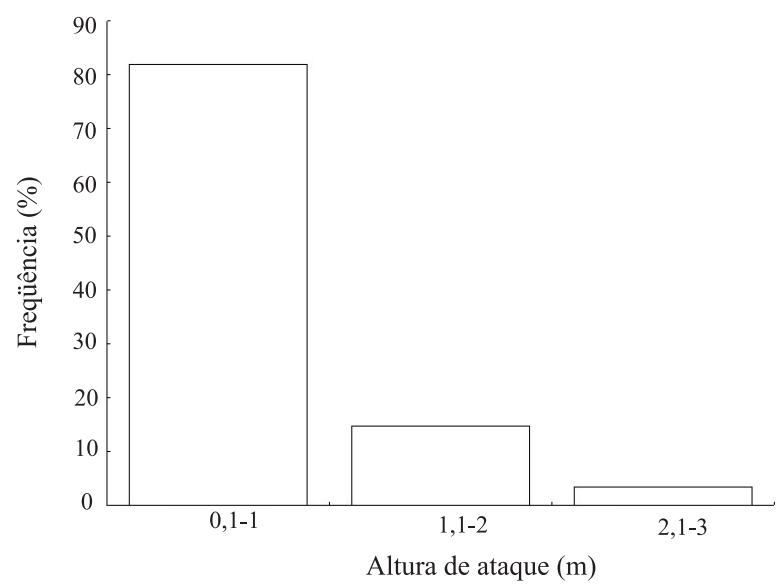

Fig. 2. Freqüência de observação de Polystictus superciliaris (Wied, 1831) em plantas de diferentes alturas utilizadas em atividades de forrageamento $(n=145)$ entre abril e dezembro de 2005 no Parque Estadual da Serra do Rola Moça, Minas Gerais, Brasil.
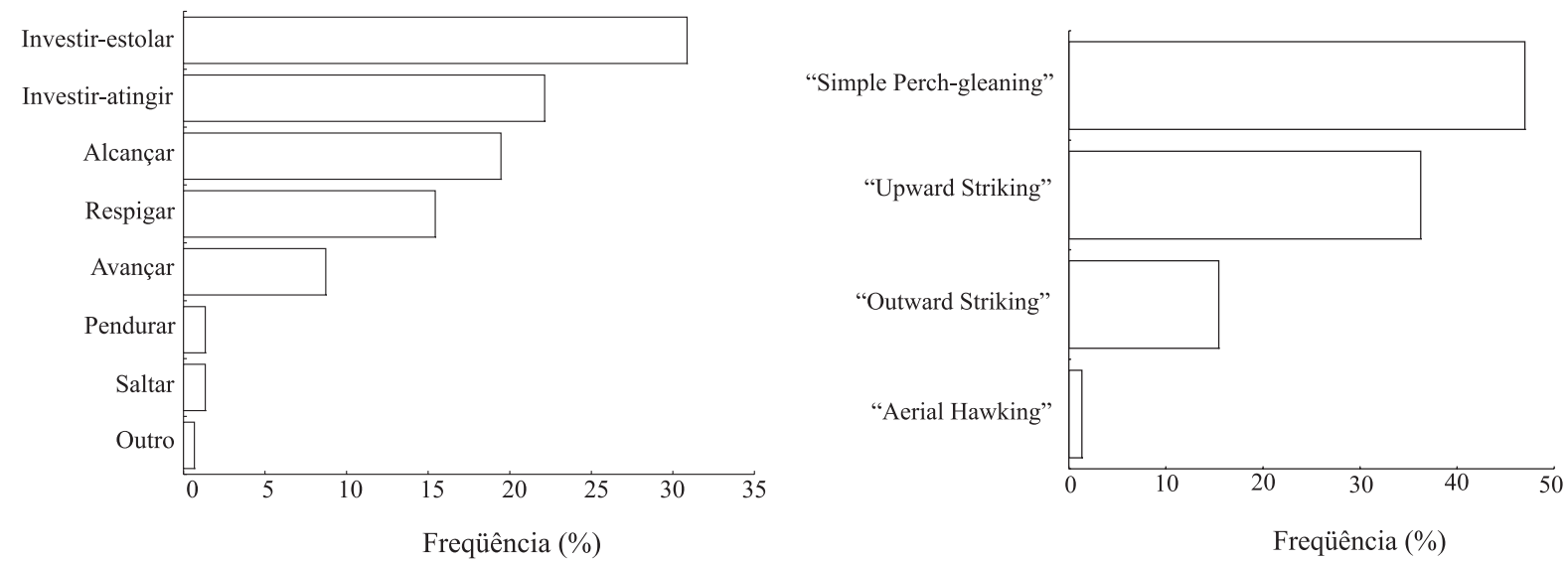

Figs. 3, 4. Freqüência de observações de Polystictus superciliaris (Wied, 1831) quanto ao comportamento de ataque entre abril e dezembro de 2005 no Parque Estadual da Serra do Rola Moça, Minas Gerais, Brasil: 3, segundo nomenclatura de Remsen \& Robinson (1990); 4, após conversão para nomenclatura proposta por FitZPATRICK (1980), para efeitos de comparação (n=149). 


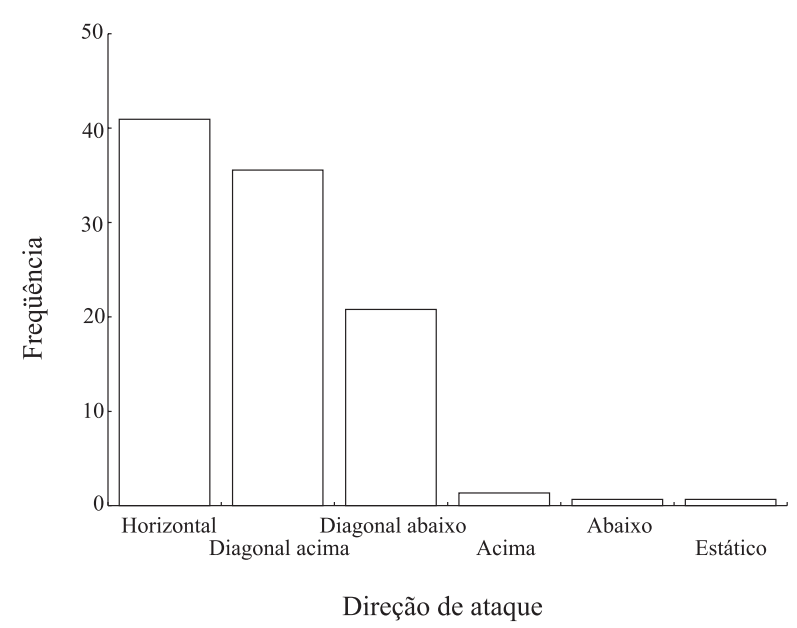

Fig. 5. Freqüência de observações de Polystictus superciliaris (Wied, 1831) quanto às direções de ataque utilizados em atividade de forrageamento $(n=149)$ no Parque Estadual da Serra do Rola Moça, Minas Gerais, Brasil

\section{DISCUSSÃO}

FitZPATRICK (1980) classificou os Tyrannidae por ele estudados como especialistas ou generalistas baseandose nas proporções dos comportamentos de forrageamento. Especialistas são aqueles que se utilizam predominantemente de um mesmo comportamento de ataque ( $>50 \%)$, enquanto os generalistas não apresentam um comportamento de ataque predominante. Devido à falta de dados, Fitzpatrick (1980) baseou-se em semelhanças morfológicas e de uso de hábitat de Culicivora caudacuta (Vieillot, 1818) para considerar o gênero Polystictus como um provável forrageador generalista em "perch-gleaning" e em "upward striking". Tal suposição foi corroborada pelos resultados do presente trabalho, que mostram que $P$. superciliaris é uma espécie que apresenta comportamento de forrageamento generalista, tanto de acordo com FitZPATRICK (1980) quanto com REMSEN \& ROBINSON (1990).

Lychnophora ericoides (Asteraceae) e Mimosa calodendron (Mimosaceae) são as espécies de plantas mais abundantes em áreas de canga couraçada no Parque Estadual da Serra do Rola Moça (D. Hoffmann, dados não publicados), o que pode explicar a maior utilização destas duas espécies como substrato de forrageamento. $\mathrm{O}$ fato de $P$. superciliaris forragear principalmente em plantas abaixo de $1 \mathrm{~m}$ de altura pode ser explicado pela baixa estatura da vegetação local, geralmente inferior a $50 \mathrm{~cm}$ (D. Hoffmann, dados não publicados).

Ridgely \& TUdoR (1994) relatam que a espécie desce ao solo ocasionalmente para se alimentar. Este comportamento raro foi observado apenas durante observações não sistematizadas, mas sempre objetivaram a recuperação de uma presa que caiu ao solo. Eventualmente, durante o forrageamento, foram observadas pequenas "escalas" em rochas, de onde os indivíduos desferiam ataques a presas localizadas em arbustos e orquídeas, sendo que em nenhuma ocasião os ataques ocorreram diretamente ao solo. Portanto, este comportamento incomum deve ser visto como uma recuperação de presa abatida e não como uma manobra de forrageamento.

As observações de aves forrageando solitariamente coincidiram com a estação reprodutiva, quando um dos indivíduos estava incubando. Tal padrão de atividade foi igualmente observado para duas outras espécies de Tyrannidae (Lopes, 2005).

Na matriz utilizada por BiRDSLEY (2002) para reconstruir a filogenia dos Tyrannidae, as espécies do gênero Pseudocolopteryx (considerado grupo irmão de Polystictus) são classificadas como especialistas "perchglean". O presente estudo demonstrou que $P$. superciliaris é uma espécie generalista, diferindo dos gêneros mais proximamente relacionados, que apresentam forrageamento do tipo "perch-glean" (BIRDSLEY, 2002).

Culicivora caudacuta, não incluído na análise de BiRDSLey (2002), é considerado por FitZPATRICK (1980) e Sick (1997) como um provável substituto geográfico de P. superciliaris. Ambas as espécies ocorrem em simpatria na área estudada, sendo $C$. caudacuta uma espécie bastante rara no local (D. Hoffmann, dados não publicados). Os dados disponíveis não permitem qualquer inferência sobre uma eventual competição entre essas duas espécies, embora as observações de campo indiquem que $C$. caudacuta se utiliza principalmente dos campos sujos, enquanto $P$. superciliaris faz uso preferencial de afloramentos de canga couraçada e campos rupestres (PARKER \& Willis, 1997; D. Hoffmann, dados não publicados).

Polystictus superciliaris exibiu uma dieta exclusivamente insetívora, fato já assinalado por VASCONCELOS (1999). Entretanto, a composição da dieta desta espécie só pôde ser determinada de maneira confiável por meio das análises de conteúdo estomacal. Tal fato se deve ao tamanho diminuto da maioria das presas consumidas, o que dificulta ou mesmo impede a sua correta identificação em campo.

Merece destaque a ausência de formigas na dieta de $P$. superciliaris, insetos que constam como um dos principais itens alimentares de muitas espécies de Tyrannidae (Poulin et al., 1994; LoPEs et al., 2005). Uma explicação simples para tal ausência seria o fato da canga couraçada apresentar uma notável escassez de formigas quando comparada à canga nodular adjacente (LOPES et $a l ., 2000)$. Tal fato provavelmente se deva às dificuldades encontradas pelas formigas em encontrar locais apropriados para construir seus ninhos, principalmente devido à limitação na disponibilidade de substratos e fendas naturais (Lopes et al., 2000). Entretanto, tal explicação não é corroborada pela análise da dieta de Embernagra longicauda Strickland, 1844, outra espécie de passeriforme que ocorre na área de estudo e que apresenta formigas como um dos seus principais itens alimentares (D. Hoffmann e colaboradores, dados não publicados). Dessa maneira, a ausência ou raridade de Formicidae na dieta de $P$. superciliaris permanece sem uma explicação adequada.

Agradecimentos. Ao Instituto Estadual de Florestas de Minas Gerais pela autorização de acesso ao Parque Estadual da Serra do Rola Moça e à direção e funcionários do parque pelo apoio e disponibilização da infra-estrutura. Ao CNPq pela bolsa de mestrado a D.H. e à FAPEMIG pela bolsa de doutorado a L. E. 
L. Ao Neotropical Bird Club, Idea Wild, Fundação O Boticário de Proteção à Natureza e CNPq (processo 473428/2004-0), que concederam auxílios de pesquisa. À Regina Macedo e a um revisor anônimo pelas críticas ao manuscrito.

\section{REFERÊNCIAS BIBLIOGRÁFICAS}

Ав'SABER, A. N. 1977. Os domínios morfoclimáticos na América do Sul. Primeira aproximação. Geomorfologia 52:1-21.

Bartholomew, G. A. 1986. The role of natural history in contemporary biology. Bioscience 36(5):324-329.

Birdsley, J. S. 2002. Phylogeny of the Tyrant Flycatchers (Tyrannidae) based on morphology and behavior. Auk 119(3):715-734.

Borror, D. J.; Triplehorn, C. A. \& Johnson, N. F. 1989. An introduction to the study of insects. 6. ed., Philadelphia, Saunder College 875p.

Drummond, G. M.; Martin, C. S.; Machado, A. B. M.; Sebaio, F. A. \& Antonini, Y. 2005. Biodiversidade em Minas Gerais. Um atlas para sua conservação. Belo Horizonte, Fundação Biodiversitas. 222p.

FitZPATRICK, J. W. 1980. Foraging behavior of Neotropical Flycatchers. Condor 82(1):43-57.

1985. Form, foraging behavior, and adaptative radiation in the Tyrannidae. In: Buckley, P. A.; Foster, M. S.; Morton, E. S.; Ridgely, R. S. \& Buckley, F. G. eds. Neotropical ornithology. Washington D. C., American Ornithologist's Union. p.447-470 (Ornithological Monographs, 36).

2004. Family Tyrannidae (Tyrant-flycatchers). In: DEL Hoyo, J.; Elliott, A. \& Christie, D. eds. Handbook of the birds of the world. Cotingas to pipits and wagtails. Barcelona, Lynx. v.9, p.170-462.

Gabriel, V. A. \& Pizo, M. A. 2005. Foraging behavior of tyrant flycatchers (Aves, Tyrannidae) in Brazil. Revista Brasileira de Zoologia 22(4):1072-1077.

Hejl, S. J.; Verner, J. \& Bell, G. W. 1990. Sequential versus initial observations in studies of avian foraging. Studies in Avian Biology 13:166-173.

inMET (Instituto Nacional de Meteorologia). 2005. Parâmetros meteorológicos de Belo Horizonte. Disponível em: <http:/ /www.inmet.gov.br/>. Acesso em: 16.01.2005.

LANYON, W. E. 1986. A phylogeny of the thirty three genera in the Empidonax assemblage of tyrant flycatchers. American Museum Novitates 2846:1-64.

1988. A phylogeny of the thirty-two genera in the Elaenia assemblage of Tyrant Flycatchers. American Museum Novitates 2914:1-57

Lopes, L. E. 2005. Dieta e comportamento de forrageamento de Suiriri affinis e S. islerorum no Cerrado do Brasil central. Iheringia, Série Zoologia, 95(4):341-345.

Lopes, L. E.; Fernandes, A. M. \& Marini, M. Â. 2005. Diet of some Atlantic Forest birds. Ararajuba 13:95-103.
Lopes, L. E.; Versieux, L. M.; Castro, C. S. \& Almeida, J. C. 2000. Diversidade de artrópodos em solos de canga na Serra da Piedade, MG. Simpósio de Ecossistemas Brasileiros: Conservação. In: Simpósio de Ecossistemas Brasileiros, 5, Vitória. Anais... Vitória, UFES. v.5, p.358-363.

Nimer, E. 1979. Climatologia no Brasil. Rio de Janeiro, Instituto Brasileiro de Geografia e Estatística, Superintendência de Recursos Naturais e Meio Ambiente. v.4, 421p.

PARKer, T. A., III. 1984. Notes on the behavior of Ramphotrigon Flycatchers. Auk 101:186-188.

Parker III, T. A. \& Willis, E. O. 1997. Notes on three tiny grassland flycatchers, with comments on the disappearance of South American fire-diversified savannas. In: REMSEN, J. V., $J_{R}$. ed. Studies in Neotropical Ornithology honoring Ted Parker. Washington D.C., American Ornithologists' Union. p.549-555 (Ornithological Monographs, 48).

Poulin, B.; Lefebvre, G. \& McNeIL, R. 1994. Diets of land birds from northeastern Venezuela. Condor 96(2):354-367.

Remsen, J. V., JR. \& Robinson, S. K. 1990. A classification scheme for foraging behavior in terrestrial habitats. Studies in Avian Biology 13:144-160.

Ridgely, R. S. \& Tudor, G. 1994. The birds of South America: the suboscine passerines. Austin, University of Texas. v. $2,814 \mathrm{p}$

RizzinI, C. T. 1979. Tratado de fitogeografia do Brasil. São Paulo, HUCITEC. 747p.

Sibley, C. G. \& Monroe JR., B. L. 1990. Distribution and taxonomy of birds of the world. New Haven, Connecticut, Yale University. $1111 \mathrm{p}$.

Sick, H. 1997. Ornitologia brasileira. Rio de Janeiro, Nova Fronteira. $862 p$.

Sкuтсн, A. F. 1960. Life histories of Central American birds, II. Families Vireonidae, Sylviidae, Turdidae, Troglodytidade, Paridae, Corvidae, Hirundinidae and Tyrannidae. Berkeley, Cooper Ornithological Society. 593p.

SмiтH, W. J. 1971. Behavioral characteristics of serpophaginine Tyrannids. Condor 73(3):259-286.

Traylor, M. A. \& FitzPatrick, J. 1982. A survey of tyrant flycatchers. Living Bird 19:7-50.

VASCONCELOS, M. F. 1999. Natural history notes and conservation of two species endemic to the Espinhaço Range, Brazil: Hyacinth Visorbearer Augastes scutatus and Grey-backed Tachuri Polystictus superciliaris. Cotinga 11:75-78.

Vasconcelos, M. F.; Maldonado-Coelho, M. \& Buzzetti, D. R. C. 2003. Range extension for the Gray-backed Tachuri (Polystictus superciliaris) and the Pale-throated Serra-Finch (Embernagra longicauda) with the revision on their geographic distribution. Ornitología Neotropical 14:477-489.

Vincent, R. C.; JACobi, C. M. \& Antonini, Y. 2002. Diversidade na adversidade. Ciência Hoje 185:64-67.

Volpato, G. H. \& Mendonç-Lima, A. 2002. Estratégias de forrageamento: proposta de termos para a língua portuguesa. Ararajuba 10:101-105 